\title{
On-Body Characterization of Textile Antennas for Biomedical Health Monitoring Systems
}

\author{
P. J. Soh ${ }^{1,2}$, G. A. E. Vandenbosch ${ }^{1}$ and D. M. M.-P. Schreurs ${ }^{1}$ \\ ${ }^{1}$ Div. ESAT-TELEMIC, Katholieke Universiteit Leuven, 3001 Leuven, Belgium \\ ${ }^{2}$ Sch. of Computer and Communication Eng., Universiti Malaysia Perlis, 02000 Perlis, Malaysia
}

\begin{abstract}
In recent years, application of textiles as sensors in wearable biomedical health monitoring is increasingly popular. Since most of such sensors require the use of a wireless data link for bio-signal transmission back to the care-givers, there exists a need to integrate wearable and flexible antennas onto such wearable monitors to ensure comfort to users. This work presents two all-textile planar inverted-F antennas (PIFAs) for such purpose, and investigates their performance in close proximity of a real human user. Besides featuring broad bandwidths, larger than requirements, both antennas also possess favorable properties, namely compact in size, conformal, and low-cost.

Index Terms - Biomedical telemetry, body sensor networks, biomedical monitoring, broadband antennas, fractal antennas, multifrequency antennas.
\end{abstract}

\section{INTRODUCTION}

The rapid advancement of wearable body sensors in biomedical applications necessitates the need for their implementation on flexible and conformal materials. These material characteristics enable such sensors to be utilized on various parts of the users' body for continuous monitoring of physiological parameters. Among such parameters include a person's blood pressure, body and skin temperature, glucose level, respiration and cardiac activities.

Recent researchers have proven that the implementation of these sensors through the use of various thin, flexible and printed structures is promising [1]. However, the use of textiles, one of the most promising materials, has been limited to the "front-end" portion of these sensors. In other words, researchers have replaced conventional materials in sensors, electrodes and probe patches using textiles to enable similar function, but with the added flexibility and user comfort. This is crucial as continuous health monitoring can only be effective once comfort to patients can be guaranteed.

Although architectures of these wearable health monitoring systems can vary depending on the desired bio-signals, they more or less consist of similar building blocks, i.e., sensors, wearable materials, actuators, power supply, wireless communication modules and links, control and processing units, software, user interface and algorithms for data processing [2]. Although not compulsory, the existence of a secure, simple and powerefficient wireless bio-signal transmission link from the user to the central processing node is preferable, requiring the development of conformal antennas for on-body mounting. However, the placement of such radiating structures at a close proximity to the human body causes degradation to its reflection and radiation property, rendering the antenna and the wireless transmission link ineffective. This will then affects crucial bio-signals transmission to the care-giver, who needs to interpret them at a fixed time-cycle. Such limitation can be effectively avoided through the antennas' on-body characterization.

Recent antenna assessment efforts have concentrated in investigating conventional, rigid antennas for radar applications [3]-[4], whereas the on-body evaluation presented here aims to complement such systems. This work evaluates two types of radiators prototyped using textile materials in proximity of a real human user. Antennas are designed based on the planar inverted-F antenna (PIFA) topology, one featuring a broadband operation, and the other, a dual-band resonance. Textiles are utilized to enable ease of integration onto a worn health monitoring system, assuming that this system also features conformal characteristics. The next section introduces these antennas' designs, dimensions, materials and radiation features. Subsequently, their reflection and radiation performance are evaluated and presented in section III in free space, before on-body assessments in section IV.

\section{ANTENNA DESIGN}

A PIFA structure generally consists of a ground plane, a radiator and a substrate sandwiched between the two elements. A $50 \Omega$ feed point and a shorting wall are also featured, with both vertical elements placed across the structure's thickness, as shown in Fig. 1. The two antennas studied featured distinct radiator shapes, enabling a broadband resonance by the first PIFA, and a dual-band resonance by the second PIFA. The first PIFA's radiator is rectangular-shaped, sized at $21 \times 19 \mathrm{~mm}^{2}$, with a substrate and ground plane sized at $50 \times 19 \mathrm{~mm}^{2}$ (referred to as the 
"Plain PIFA (PPIFA)") [5], whereas the second PIFA's radiator is triangular in shape, sized at $34 \times 24 \mathrm{~mm}^{2}$, incorporated with a similarly-shaped, triangular slot sized at $17 \times 12 \mathrm{~mm}^{2}$ (referred to as "Fractal PIFA (FPIFA)") [6]. This FPIFA's substrate and ground plane are similarly sized at $44 \times 34 \mathrm{~mm}^{2}$ which enables the conception of the fractal topology, and permitting the realization of a dualresonance structure. Dimension estimation of both antennas is carried out according to procedures detailed in [7] for PPIFA and [8] for FPIFA. Both antennas are designed to resonate within the $2.45 \mathrm{GHz}$ Industrial, Scientific and Medical (ISM) band, while the FPIFA is intended for operation in the 2.4/5.2 GHz Wireless Local Area Network (WLAN) band.

ShieldIt conductive textile from LessEMF Inc. is utilized as both antennas' radiating elements and ground planes, while $6 \mathrm{~mm}$-thick pieces of felt are used as their substrate. Conductive epoxy model 8331 from MG Chemicals Inc. is used to enable the $50 \Omega$ SMA connector to textile interconnection without soldering, as this risks damage to the textile. Simulations are carried out using CST Microwave Office solver, while the manual fabrication procedure detailed in [7].

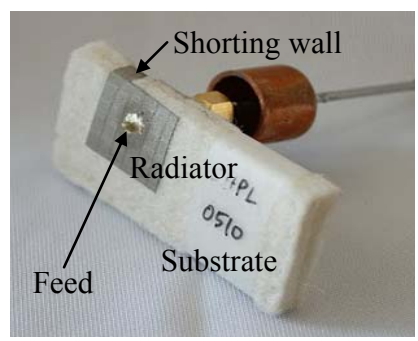

(a)

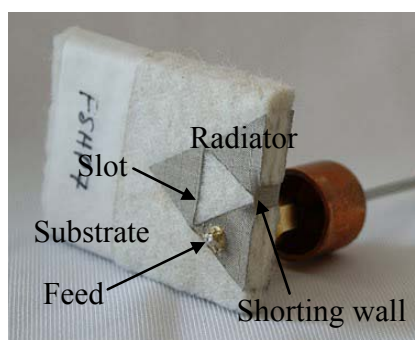

(b)
Fig. 1. Structure of the fabricated antenna prototypes: (a) PPIFA and (b) FPIFA; and their major constituting elements.

\section{Free Space EVALUATION}

The antennas' reflection coefficients $\left(\mathrm{S}_{11}\right)$ are measured using a Vector Network Analyzer, while their radiation performance are assessed in an anechoic chamber. The minimum acceptable $\mathrm{S}_{11}$ is $-10 \mathrm{~dB}$, indicating a maximum RF power reflection of $10 \%$. The difference between these $\mathrm{S}_{11}$ borders is taken as their bandwidths $(B W)$, while center frequency $\left(f_{\mathrm{c}}\right)$ is located at their respective $B W$ center. PPIFA measurements indicated a satisfactory performance, with a broadband operation between 1.98 $\mathrm{GHz}$ and $3.26 \mathrm{GHz}$, which translates to a $B W$ of $1.28 \mathrm{GHz}$ in free space. The FPIFA operation in free space, meanwhile, indicated measured $B W$ of $325 \mathrm{MHz}$ and 563 $\mathrm{MHz}$ in the lower and upper band, respectively. Although variations are observed compared to simulations, both measured antennas' $B W$ is sufficient to fulfill requirements for bio-signals transmission at both frequencies. On top of that, this additional $B W$ also caters for variations when operated on body, and will be explained in the next section.

Radiation characteristics of the antenna in free space indicated linear polarization, radiating uni-directionally outwards from the body at the $\phi=90^{\circ}$ plane, while featuring an omni-directional $\phi=0^{\circ}$ cut, enabled by the full ground plane featured in this PIFA topology. This implies a minimal amount of back radiation generated, and the PIFAs are most suitable to be used as the transmitting/receiving nodes, radiating outwards from the body. The results are plotted in Fig. 3. To simplify analysis and enable direct comparison, only $2.45 \mathrm{GHz}$ radiation patterns are compared.

TABLE I

Summary of Free Space and On Body Measurements

\begin{tabular}{|l|c|c|c|c|c|}
\hline Ant Type/OB location & FS & $\mathrm{CH}$ & $\mathrm{CV}$ & $\mathrm{BH}$ & $\mathrm{BV}$ \\
\hline PPIFA $B W(\mathrm{MHz})$ & 1280 & 1160 & 990 & 1230 & 1500 \\
\hline PPIFA $f_{\mathrm{c}}(\mathrm{GHz})$ & 2.62 & 2.53 & 2.47 & 2.60 & 2.62 \\
\hline PPIFA $\eta_{\text {rad }}(\%)$ Sim & 80.9 & 32.9 & 38.9 & 59.6 & 56.2 \\
\hline PPIFA Gain $(\mathrm{dB})$ & 1.53 & & & & \\
\hline FPIFA $B W(\mathrm{~L})(\mathrm{MHz})$ & 325 & 275 & 350 & 287 & 300 \\
\hline FPIFA $B W(\mathrm{U})(\mathrm{MHz})$ & 563 & 544 & 538 & 519 & 569 \\
\hline FPIFA $f_{\mathrm{c}}(\mathrm{L})(\mathrm{GHz})$ & 2.14 & 2.09 & 2.14 & 2.09 & 2.09 \\
\hline FPIFA $f_{\mathrm{c}}(\mathrm{U})(\mathrm{GHz})$ & 5.28 & 5.21 & 5.12 & 5.20 & 5.15 \\
\hline FPIFA $\eta_{\text {rad }}(\%)(\mathrm{L})$ Sim & 80.3 & 37.3 & & 56.6 & \\
\hline FPIFA $\eta_{\text {rad }}(\%)(\mathrm{U})$ Sim & 79.3 & 43.8 & & 52.9 & \\
\hline FPIFA Gain $(\mathrm{dB})$ & 1.80 & & & & \\
\hline
\end{tabular}

Legend: FS-free space, CH-chest horizontal, CV-chest vertical, BH-back horizontal, BV-back vertical, (L)-lower band, (U)-upper band.

\section{ON-BODY ASSESSMENTS}

On-body (OB) performance assessment is carried out on a male human volunteer, of $1.78 \mathrm{~m}$ height and $90 \mathrm{~kg}$. To maintain a consistent evaluation location and avoid measurement inaccuracies induced by cable movements, two similar $40 \mathrm{~cm}$ cables are routed and sewn onto the reverse side of a fleece jacket. Note that this jacket is only introduced to facilitate accurate antennas' characterization. Evaluations are carried out on two potential locations on the human upper torso, i.e., on the chest and back, where vital bio-signals are most likely to be collected from. Antennas under test (AUTs) are evaluated both vertically and horizontally on each location to determine the most suitable orientation (denoted as $\mathrm{CH}$ and $\mathrm{CV}$ for chest, and $\mathrm{BH}$ and $\mathrm{BV}$ for back). A $10.3 \mathrm{~mm}$ felt spacer is attached to the ground plane of both AUTs to emulate a realistic spacing where the health monitoring system is to be placed. 


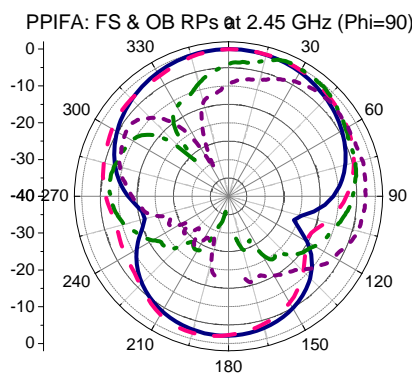

(a)

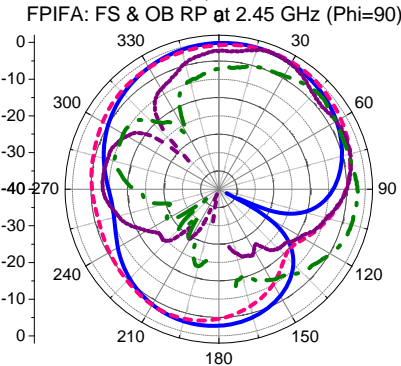

(c)

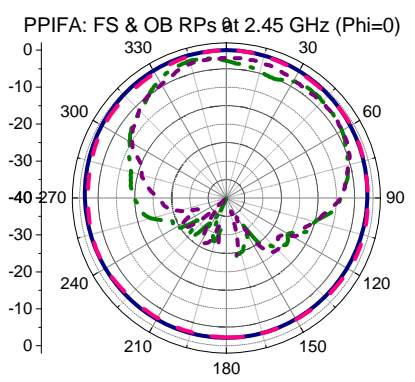

(b)

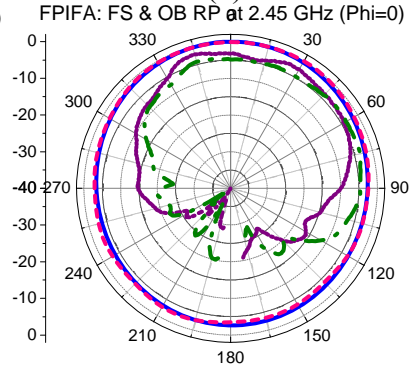

(d)

Fig. 2. Free space and on-body simulated and measured radiation patterns at $2.45 \mathrm{GHz}$ : (a) FPIFA at $\phi=0^{\circ}$, (b) PPIFA at $\phi=0^{\circ}$, (c) FPIFA at $\phi=90^{\circ}$, (d) PPIFA at $\phi=90^{\circ}$. Legend: (Blue, Solid): Simulated in FS; (Pink, Dash): Measured in FS; (Green, Dash Dot): Measured for back; (Purple, Short Dash): Measured for chest.

The $\mathrm{S}_{11}$ changes for both antennas are first analyzed and summarized in Table I. As observed, the $B W$ is generally degraded for both antennas when placed on the chest, regardless of antenna orientation. For back placement, however, a different behavior is observed. Similar to $\mathrm{CH}$ and $\mathrm{CV}$, the decreasing $B W$ is also noticeable when the PIFAs are placed using configuration $\mathrm{BH}$. BV, however, slightly increased, especially in the lower bands for both PIFAs. This indicates a strong influence on the antenna's Q, causing a PPIFA $B W$ expansion of up to $220 \mathrm{MHz}$ relative to free space. $f_{\mathrm{c}}$, is generally moving down the frequency scale when placed anywhere on the body. Nonetheless, $f_{\mathrm{c}}$ for $\mathrm{BH}$ and $\mathrm{BV}$ orientations are on average less affected by on-body placement.

Analyzing the measured on-body patterns, the antennas' back radiation is seen in Fig. 2 to be affected by body absorption. However, at this plane, forward radiation profile is maintained for both PIFAs, indicating a similar half-power beamwidth (HPBW) against free space. This also means that direction-wise, the transmission of biosignals at the outwards direction from the body is minimally affected. It can be seen that there exists a null at ca. $330^{\circ}$. This is mainly due to the strong antenna-to-body coupling at both PIFAs' radiating edges due to their small ground planes.

\section{CONCLUSIONS}

A pair of antennas based on a similar PIFA topology and distinct radiator shapes have been designed, prototyped and evaluated both in free space and on body. Besides their excellent free space performance in terms of reflection coefficient and radiation characteristics, both PIFAs have been validated to generate more bandwidth than required for operation in the ISM and $2.4 / 5.2 \mathrm{GHz}$ WLAN bands. This technique of generating extra bandwidth is seen effective and simple in catering for any degradation during operation in proximity of a human user. This investigation has also proven that such antennas are practical and readily available for use for wireless data transmission in wearable health monitoring systems.

\section{ACKNOWLEDGEMENT}

The authors wish to acknowledge the assistance and of Wee Fwen Hoon and Dr. Mohd Fareq Malek for the Hugo simulations.

\section{REFERENCES}

[1] M. Di Rienzo, P. Meriggi, F. Rizzo, P. Castiglioni, C. Lombardi, M. Ferratini, and G. Parati, "Textile technology for the vital signs monitoring in telemedicine and extreme environments," IEEE Trans on Information Tech. in Biomedicine, vol. 14, no. 3, pp. 711-717, May 2010.

[2] A. Pantelopoulos and N. G. Bourbakis, "A survey on wearable sensor-based systems for health monitoring and prognosis," IEEE Trans. on Systems, Man and Cybernetics, Part C, vol. 40, no. 1, pp. 1-12, Jan 2010.

[3] C. Gu, Z. Salmani, H. Zhang, C. Li, "Antenna array technology for radar respiration measurement in motionadaptive lung cancer radiotherapy," in Proc. 2012 IEEE Top. Conf. on Biomedical Wireless Tech., Network, and Sensing Syst., pp. 21-24.

[4] V. Das, A. Boothby, R. Hwang, T. Nguyen, J. Lopez, D. Y. C. Lie, "Antenna evaluation of a non-contact vital signs sensor for continuous heart and respiration rate monitoring," in Proc. 2012 IEEE Top. Conf. on Biomedical Wireless Tech., Network, and Sensing Syst., pp. 13-16.

[5] P. J. Soh, S. J. Boyes, G. A. E. Vandenbosch, Y. Huang, S.L Ooi, "On-body Characterization of Dual-Band AllTextile PIFA," Progress in Electromagnetic Research, vol. 129, pp. 517-539, 2012.

[6] P. J. Soh, G.A.E Vandenbosch, V. Volski, H. M. R. Nurul, "Characterization of a Simple Broadband Textile Planar Inverted-F (PIFA) for On-Body Communications," ICECom 2010 Conf. Proc., pp. 1-4, 20-23 Sept. 2010

[7] P. J. Soh, G. A. E. Vandenbosch, S. L. Ooi, N. H. M. Rais, "Design of a broadband all-textile slotted PIFA," IEEE Trans. on Antennas and Propagation, vol. 60, no. 1, pp. 379-384, Jan 2012.

[8] P. J. Soh, G. A. E. Vandenbosch, S. L. Ooi, M. R. N. Husna, "Wearable dual-band Sierpinski fractal PIFA using conductive textiles," Electronics Letters, vol. 47, no. 6, pp. 365-367, Mar 2011. 